\title{
Microparticles (CD146) and Arterial Stiffness Versus Carotid Intima Media Thickness as an Early Predictors of Vascular Affection in Systemic Lupus Patients
}

\author{
Sahar NASSEF, ${ }^{1}$ Hala El GUINDEY, ${ }^{1}$ Mary FAWZY,,${ }^{1}$ Amal NASSER,${ }^{2}$ Rasha REFFAI, ${ }^{3}$ Doa SHEMIY ${ }^{1}$ \\ ${ }^{1}$ Department of Internal Medicine, Cairo University, Cairo, Egypt \\ ${ }^{2}$ Department of Clinical Pathology, Cairo University, Cairo, Egypt \\ ${ }^{3}$ Department of Rheumatology, Cairo University, Cairo, Egypt
}

\begin{abstract}
Objectives: This study aims to evaluate cluster of differentiation 146 (CD146) and pulse wave velocity (PWV) as non-invasive methods for prediction of early vascular affection in systemic lupus erythematosus (SLE) patients without symptoms of vascular disease, to detect the outcome and reproducibility of these methods, and to correlate CD146 and PWV with lipid profile, intima media thickness (IMT), and ankle brachial index.

Patients and methods: Thirty female SLE patients (mean age $26.6 \pm 6.6$ years; range 15 to 35 years) fulfilling the American College of Rheumatology 1997 revised criteria for SLE classification, and 15 age and sex matched healthy controls were included. All participants were performed full clinical assessments including measurement of Systemic Lupus Erythematosus Disease Activity Index, lipid profile, CD146, carotid IMT, PWV, and rise time as an indication of how fast the waveform rises.

Results: Cluster of differentiation 146 levels were elevated in patients with SLE compared to controls $(p<0.001)$. There was a statistically significant difference between patients and controls in the femoral, lower thigh, and ankle rise time. There was a statistically significant correlation between IMT and ages of patients, Systemic Lupus Erythematosus Disease Activity Index, and brachial-below knee PWV, while there was no correlation between IMT and disease duration, lipid profile, brachial-femoral PWV, and brachial-ankle PWV. There was statistically significant correlations between brachial-femoral PWV and serum cholesterol level, and between brachial-ankle PWV and low density lipoprotein cholesterol.

Conclusion: Our results showed that SLE vascular affection is more pronounced in small arteries. Also, elevated CD146 and brachial-femoral PWV are useful early markers of vascular affection in SLE as well as rise time may be a marker for arterial stiffness.

Keywords: Arterial stiffness; cluster of differentiation 146; pulse wave velocity; systemic lupus erythematosus.
\end{abstract}

Systemic lupus erythematosus (SLE) is an inflammatory disease caused by autoimmune dysregulation, which mainly affects young females, usually free from atherosclerosis. Accelerated atherosclerosis is a well-established complication of SLE and it cannot be explained by Framingham risk factors alone, and has been attributed to complex interactions between traditional risk factors and factors associated with the disease per se, or its treatment. ${ }^{1}$ The prevalence of cardiovascular disease ranges from 6 to $10 \%$ in individuals with SLE, and the risk of developing cardiovascular disease is 4 to 8 times higher than in normal population. ${ }^{2}$ Moreover, acute myocardial infarction is reported as a cause of death in 3 to $25 \%$ of SLE patients in different surveys. ${ }^{3}$ Patients with SLE have increased vascular morbidity and mortality. Several established vascular risk factors are more prevalent in this population but cannot fully explain the reported excess atherosclerotic burden. Emerging vascular risk factors may also contribute to the increased vascular risk in these patients although the evidence is limited and often conflicting. SLE-specific risk factors also play a role in the pathogenesis of atherosclerosis. Given the multifactorial etiology of vascular disease in SLE, an integrated index of risk may be useful in the management of these patients.

Received: July 13, 2015 Accepted: August 24, 2015 Published online: October 03, 2015

Correspondence: Mary Fawzy, MD. Department of Internal Medicine, Cairo University, 12311 Cairo, Egypt.

Tel:002-01006196952 e-mail:drmarywadie@yahoo.com

O2016 Turkish League Against Rheumatism. All rights reserved. 
Arterial stiffness possibly represents such an index and accumulating data suggest an increased prevalence of arterial stiffness in SLE. Many factors play a role in the loss of arterial elasticity in this population, including both emerging and established vascular risk factors. Arterial stiffness may emerge as a useful index for risk stratification in SLE and has the potential to guide therapeutic decisions in these patients. ${ }^{4}$ Among the different methods of evaluating arterial stiffness, the most widely used in the literature is pulse wave velocity (PWV), specifically in the area running from the aortic arch or common carotid artery to the common femoral artery. Typically, the pulse wave is detected by pressure transducers or arterial tonometry. ${ }^{5}$ Tanaka et al. ${ }^{6}$ concluded that carotidfemoral PWV and brachial-ankle PWV are indices of arterial stiffness that exhibit similar extent of associations with cardiovascular disease risk factors and clinical events.

Cluster of differentiation 146 (CD146) also known as the melanoma cell adhesion molecule or cell surface glycoprotein MUC18, is a cell adhesion molecule currently used as a marker for endothelial cell lineage and is involved in the control of cell cohesion, vascular permeability, and monocyte transmigration. ${ }^{7}$ Its function is still poorly understood, but evidence points to it being part of the endothelial junction associated with the actin cytoskeleton. The positive correlation between CD146 and plasma intracellular adhesion molecule and von Willebrand factor levels supports its role as a marker of endothelial dysfunction. ${ }^{8}$

In this study, we aimed to evaluate CD146 and PWV as non-invasive methods for prediction of early vascular affection in SLE patients without symptoms of vascular disease, to detect the outcome and reproducibility of these methods, and to correlate CD146 and PWV with lipid profile, intima media thickness (IMT), and ankle brachial index.

\section{PATIENTS AND METHODS}

A total of 30 female SLE patients (mean age $26.6 \pm 6.6$ years; range 15 to 35 years) who fulfilled the 1997 American College of Rheumatology revised criteria for classification of $\mathrm{SLE}^{9}$ with no history of cardiovascular disease were included in this randomized controlled study. The patients were selected from patients admitted to internal medicine department of Kasr Al-Ainy Hospital, as well as patients from the rheumatology \& immunology outpatient clinic, internal medicine department, Cairo University. The study also included 10 age and sex matched apparently healthy volunteers, with no history of autoimmune diseases, as a control group. Local institutional research board approval as well as written informed consents were obtained from all the subjects prior to participation in the study.

Systemic lupus erythematosus patients with any cardiovascular, cerebrovascular or peripheral vascular disease, diabetes mellitus or smoking were excluded.

All subjects were subjected to full history taking, detailed clinical examination including systemic as well as rheumatological examinations with special concern regarding mucocutaneous, joint, renal and neuropsychiatric manifestations, also vascular disorders including arterial and venous disorders; routine laboratory investigations including complete blood picture, erythrocyte sedimentation rate, urine analysis, 24 hour urinary proteins, liver function tests, kidney function tests and CD146 by flow cytometry.

Patients were also subjected to lipid profile tests for cholesterol and triglycerides, and immunological profile tests for antinuclear antibodies, DNA double-stranded antibodies, serum complement 3 (C3), complement C4, lupus anticoagulant, and anticardiolipin.

Two milliliter of venous blood was obtained and preserved in ethylenediaminetetraacetic acid vacutainers. Cells were incubated with antiCD146-FITC (Becton-Dickinson) together with anti-CD4-PE (Becton-Dickinson). Fluorescence intensity was determined using the FACScan from Becton-Dickinson.

Arterial stiffness of patients and controls was assessed noninvasively. Subjects were refrained from eating or drinking coffee, tea, or alcohol at least 12 hours prior to the study. To ensure the stability of measurement, arterial stiffness test was performed in the supine position after resting for 10 minutes in a quiet, temperature controlled room $\left(22-24{ }^{\circ} \mathrm{C}\right)$ in the early morning hours. Blood pressure was recorded in the right arm using an automatic blood pressure monitor. 
We used Vasoguard machine (NicoletVasoguard, model p84, VIASYS Healthcare, USA) (Figure 1) to perform a full range of physiological tests, including automated examinations for lower and upper segmental arterial studies. We applied the cuffs and photoplethysmography (PPG) sensors to the patient, and connected the cuffs and PPG sensors to the instrument according to the color coding. We attached the cuff to the appropriate air hose (Figure 2). Afterwards, we attached the PPG sensors to the fingers and toes. We administered the Doppler probe $(8 \mathrm{MHz})$ to the skin at an angle of 45 to 60 degrees to the flow direction, using a water-soluble ultrasound contact gel. We located an arterial signal in the arm distal to the cuff (brachial, radial, or ulnar) and adjusted the Doppler waveform gain and scale to optimize the spectral display. We used the space bar to inflate the cuff to the target pressure. We listened for the return of the Doppler signal and observed the spectral display. When the pulse returned, we pressed the space bar to freeze. We performed the same technique to assess the segmental pressures of the lower limbs by choosing segment. The segmental pressures screen was used for measuring the systolic pressures at the sites of the various pressure cuffs. Ankle and segmental brachial pressures were calculated automatically.

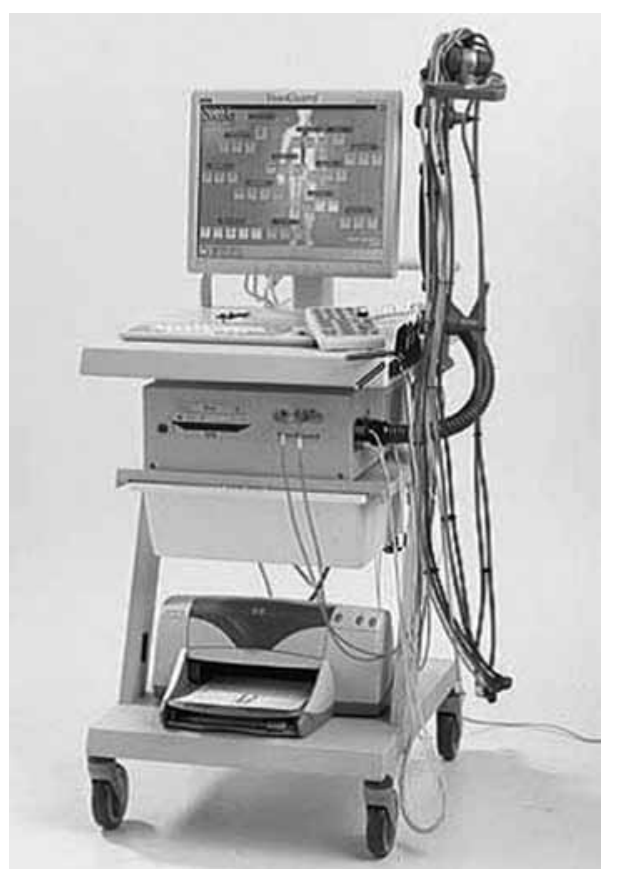

Figure 1. Vasoguard machine.
There are several calculations automatically performed using the waveform averaging function including rise time (RT), transit time. RT is an indication of how fast the waveform rises and is calculated using the entire waveform height. RT is calculated from the onset of systole (beginning of waveform upstroke) to peak systole and is the preferred method. RT is expressed in milliseconds.

Transit time was determined once all data were acquired on all channels by identifying a zero time at the beginning of the brachial pulse volume recording waveform. The time difference between start of the brachial wave and start of the waveform at another site is the transit time. The distance between the heart and the arm is essentially the same as between the heart and the aorto-iliac segment. On the report screen and the printout, the transit time was stated as the difference between cuff sites and the right brachial site. We measured the distance from the lower end of the brachial cuff to the lower end of the pressure cuffs of the lower limb by a ruler tap. We used the equation distance/transit time (time delay) to calculate the PWV.

Photoplethysmography sensors were placed on the toes to monitor the returning pulse for detecting segmental pressure. PPG sensors allow bilateral pressures to be obtained simultaneously. First, we applied the blood pressure cuffs to the arms and legs, as described earlier, and connected the hoses.

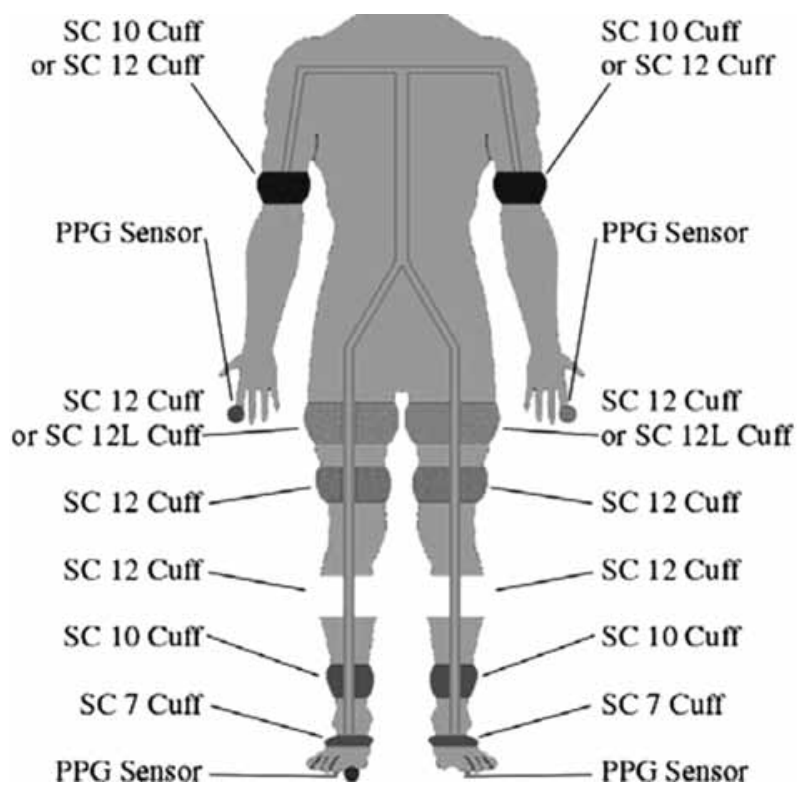

Figure 2. Cuff size and place of insertion. 
The red PPG transducer was put in the right index finger and the yellow PPG sensor in the left index finger using the PPG clips. The cuffs were inflated using the space bar until waves disappeared and then deflated until wave returns. Segmental brachial pressures were calculated automatically.

Duplex and color coded Doppler examination of both carotid arteries were performed using an ultrasound machine (Acuson Antares; Siemens, Erlangen, Germany) with a $7.5 \mathrm{MHz}$ transducer. All patients were performed Doppler by $\mathrm{B}$ (brightness)-mode grey scale, color, spectral Doppler techniques. We assessed the carotid IMT and any plaques were recorded (Figure 3).

\section{Statistical analysis}

Analysis of data was performed using SPSS for Windows version 12.0 software program (SPSS Inc., Chicago, IL, USA). Quantitative variables were described as mean \pm standard deviation and range, qualitative variables were described as number and percentage. Chi-square test was used to compare qualitative variables and Spearman correlation was used to rank positively or inversely variables versus each other in the same group. One way ANOVA test was used to compare more than two groups as regard to quantitative parametric variable, while Kruskal-

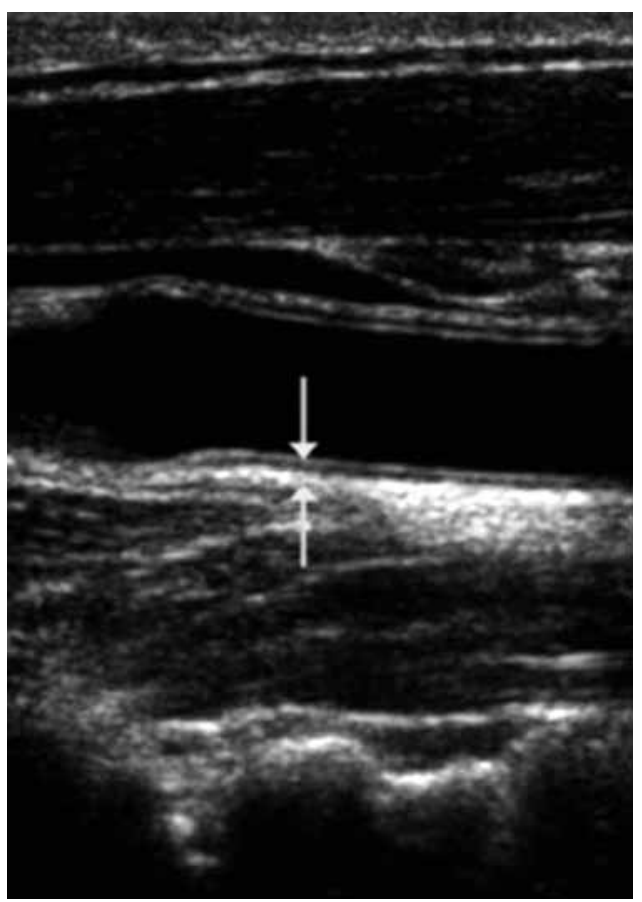

Figure 3. Carotid intima media thickness.
Wallis test was used instead of one-way ANOVA in nonparametric data (standard deviation $>50 \%$ mean). Unpaired t-test was used to compare two groups as regard to parametric variables and Mann-Whitney test was used instead of unpaired t-test in nonparametric data.

\section{RESULTS}

Disease duration of patients was $1.5 \pm 1$ years. Systemic lupus erythematosus Disease Activity Index (SLEDAI) ranging from $0-9$ had a mean score of $4.2 \pm 2$. Different clinical presentations were shown in Figure 4.

Of SLE patients, 20 had dyslipidemia. There was a statistically significant difference between patients and controls in terms of serum cholesterol, serum low density lipoprotein cholesterol (LDLc) $(p<0.001)$, and CD146 levels $(p<0.001)$ (Table 1).

There was no statistically significant difference in IMT between SLE patients and controls (Table 1), and also between dyslipidemic and non-dyslipidemic patients (Table 2). There was no statistically significant correlation between carotid IMT and serum cholesterol, serum triglyceride, LDLc, and high density lipoprotein cholesterol levels (Table 3).

There was a statistically significant difference between patients and controls in terms of femoral, lower thigh, and ankle RT $(\mathrm{p}<0.05)$ (Table 1$)$; however, there was no statistically significant difference between patients and control in terms of lower limb PWV at different segmental lower limb pressures $(p>0.05)$ (Table 1$)$.

There was a statistically significant correlation between IMT and ages of our patients, SLEDAI scores, urine albumin and albumin/creatinine

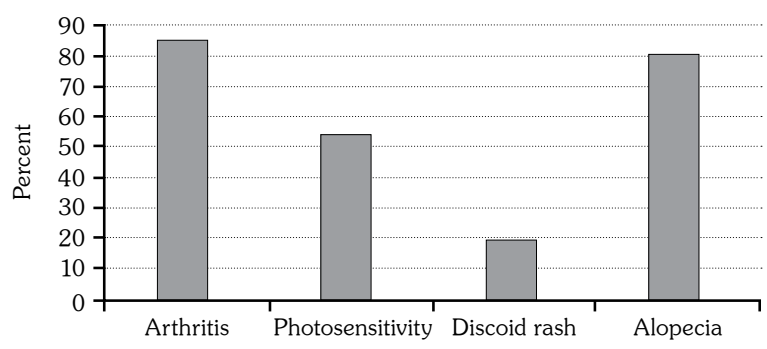

Figure 4. Different systemic lupus erythematosus presentations in our patients. 


\begin{tabular}{|c|c|c|c|c|}
\hline \multirow[t]{2}{*}{ Variables } & \multirow{2}{*}{$\frac{\text { Controls }(\mathrm{n}=10)}{\text { Mean } \pm \mathrm{SD}}$} & \multirow{2}{*}{$\frac{\text { Patients }(\mathrm{n}=30)}{\text { Mean } \pm \mathrm{SD}}$} & \multirow[t]{2}{*}{$p$} & \multirow[t]{2}{*}{$\mathrm{t}$} \\
\hline & & & & \\
\hline Systolic blood pressure & $101 \pm 9$ & $107.5 \pm 13$ & $>0.05 \mathrm{NS}$ & 1.4 \\
\hline Diastolic blood pressure & $67.5 \pm 7$ & $66.5 \pm 8$ & $>0.05 \mathrm{NS}$ & 0.6 \\
\hline Erythrocyte sedimentation rate & $11 \pm 2$ & $60.1 \pm 12$ & $<0.001 \mathrm{HS}$ & 4.7 \\
\hline Cholesterol & $120 \pm 28$ & $179 \pm 49$ & $<0.001 \mathrm{HS}$ & 3.5 \\
\hline High density lipoprotein & $36.9 \pm 7.8$ & $45.9 \pm 20$ & $>0.05 \mathrm{NS}$ & 1.3 \\
\hline Low density lipoprotein & $61.2 \pm 23$ & $107 \pm 45$ & $<0.001 \mathrm{HS}$ & 3 \\
\hline Triglycerides & $102.3 \pm 35$ & $157 \pm 60$ & $>0.05 \mathrm{NS}$ & 1.7 \\
\hline Cluster of differentiation 146 & $2.2 \pm 0.7$ & $4.2 \pm 1.1$ & $<0.001 \mathrm{HS}$ & \\
\hline Pressure index femoral & $1.4 \pm 0.2$ & $1.3 \pm 0.2$ & $>0.05$ & 0.9 \\
\hline Photoplethysmography femoral & $1.2 \pm 0.3$ & $1.2 \pm 0.2$ & $<0.005 \mathrm{HS}$ & 0.3 \\
\hline Pressure index lower thigh & $1.28 \pm 0.08$ & $1.34 \pm 0.17$ & $>0.05$ & 0.6 \\
\hline Pressure index lower thigh & $1.34 \pm 0.17$ & $1.28 \pm 0.08$ & $>0.05$ & 0.6 \\
\hline Photoplethysmography lower thigh & $1.11 \pm 0.2$ & $1.12 \pm 0.2$ & $>0.05$ & 0.3 \\
\hline Pressure index below knee & $1.20 \pm 0.08$ & $1.15 \pm 0.08$ & $>0.05$ & 0.9 \\
\hline Mean pressure index ankle & $1.14 \pm 0.06$ & $1.13 \pm 0.09$ & $>0.05$ & 1.1 \\
\hline Photoplethysmography below knee & $0.98 \pm 0.08$ & $0.99 \pm 0.09$ & $>0.05$ & 0.4 \\
\hline Photoplethysmography ankle & $0.97 \pm 0.11$ & $0.99 \pm 0.12$ & $>0.05$ & 0.7 \\
\hline Brachial-femoral pulse wave velocity & $7.6 \pm 1.2$ & $8.5 \pm 1.2$ & $>0.05$ & 1.6 \\
\hline Brachial-lower thigh pulse wave velocity & $7 \pm 1.5$ & $8 \pm 1.6$ & $>0.05$ & 1.2 \\
\hline Brachial-below knee pulse wave velocity & $6.7 \pm 2$ & $7.1 \pm 0.9$ & $>0.05$ & 1.3 \\
\hline Brachial-ankle pulse wave velocity & $6.7 \pm 0.9$ & $6.7 \pm 1.4$ & $>0.05$ & 0.6 \\
\hline Brachial rise time & $27.9 \pm 2.6$ & $26.4 \pm 4.9$ & $>0.05$ & 0.8 \\
\hline Femoral rise time & $45.7 \pm 7.4$ & $39.3 \pm 7.2$ & $<0.05$ & 2.3 \\
\hline Lower thigh rise time & $46.7 \pm 8$ & $41 \pm 7$ & $<0.05$ & 2 \\
\hline Below knee rise time & $37.6 \pm 5$ & $34.8 \pm 6.2$ & $>0.05$ & 1.5 \\
\hline Ankle rise time & $36.9 \pm 8$ & $31.9 \pm 6$ & $<0.001 \mathrm{HS}$ & 4.3 \\
\hline Intima media thickness & $0.050 \pm 0.007$ & $0.053 \pm 0.008$ & $>0.05$ & 0.2 \\
\hline
\end{tabular}

ratio (A/C ratio) (Table 4), and brachial-below knee PWV $(p<0.005)$. There was no statistically significant correlation between IMT and disease duration, lipid profile, brachial-femoral PWV, and brachial-ankle PWV (Table 3).

There was a statistically significant correlation between brachial-femoral PWV and serum LDLc, C3 A/C ratio, and SLEDAI scores. A statistically significant correlation was detected between brachial-lower thigh PWV and brachialbelow knee PWV with LDL, serum triglyceride, and SLEDAI scores. There was a statistically significant correlation between ankle PWV, A/C ratio, and C3 ( $p<0.005)$ (Table 3).
A statistically significant correlation was shown between systolic blood pressure and brachiallower thigh, brachial-below knee, and brachialankle PWV ( $\mathrm{p}<0.05)$ (Table 5).

\section{DISCUSSION}

Systemic lupus erythematosus is a chronic multisystem autoimmune disease with a broad range of clinical manifestations. The disease pathogenesis is attributed to circulating antinuclear autoantibodies against a variety of nuclear antigens (including double-stranded deoxyribonucleic

Table 2. Comparison between dyslipidemic and non-dyslipidemic patients as regards to cluster of differentiation 146 , intima media thickness, and brachial-femoral pulse wave velocity

\begin{tabular}{|c|c|c|c|c|c|}
\hline \multirow[t]{2}{*}{ Items of comparison } & \multicolumn{2}{|c|}{ Non-dyslipidemic group $(n=10)$} & \multicolumn{2}{|c|}{ Dyslipidemic group $(n=10)$} & \multirow[b]{2}{*}{$p$} \\
\hline & Mean \pm SD & Range & Mean \pm SD & Range & \\
\hline Cluster of differentiation 146 & $3.5 \pm 1.91$ & $0.5-8.3$ & $3.6 \pm 1.5$ & $0.8-7.7$ & $>0.05 \mathrm{NS}$ \\
\hline Intima media thickness & $0.053 \pm 0.005$ & $0.05-0.06$ & $0.054 \pm 0.009$ & 0.04-0.08 & $>0.05 \mathrm{NS}$ \\
\hline Brachial femoral pulse wave velocity & $8.23 \pm 1.43$ & $5.80-10.08$ & $8.63 \pm 1.48$ & $5.97-11.82$ & $>0.05 \mathrm{NS}$ \\
\hline
\end{tabular}


Table 3. Correlation between pulse wave velocity versus other variables

\begin{tabular}{|c|c|c|c|c|}
\hline \multirow[t]{2}{*}{ Variables } & \multicolumn{4}{|c|}{ Pulse wave velocity } \\
\hline & Brachio-femoral & Brachial-lower thigh & Brachial below knee & Brachial-ankle \\
\hline Age & -0.03 & 0.11 & 0.03 & 0.09 \\
\hline Systemic lupus erythematosus disease activity index & 0.13 & 0.22 & -0.01 & -0.01 \\
\hline Disease duration & 0.12 & 0.09 & 0.18 & 0.23 \\
\hline Erythrocyte sedimentation rate & -0.08 & 0.03 & 0.15 & 0.23 \\
\hline Cholesterol & 0.22 & 0.12 & 0.17 & 0.38 \\
\hline High density lipoprotein & 0.17 & -0.10 & -0.12 & -0.13 \\
\hline Low density lipoprotein & 0.37 & 0.03 & -0.03 & 0.012 \\
\hline Triglyceride & 0.08 & -0.02 & 0.02 & 0.08 \\
\hline Complement 3 & -0.012 & -0.18 & -0.01 & -0.02 \\
\hline Complement 4 & -0.12 & -0.09 & -0.02 & -0.13 \\
\hline Albumin/creatinine ratio & -0.01 & 0.20 & 0.18 & 0.02 \\
\hline Brachial rise time & -0.02 & 0.11 & 0.22 & 0.11 \\
\hline Femoral rise time & -0.10 & 0.02 & 0.17 & 0.10 \\
\hline Ankle rise time & 0.04 & 0.21 & -0.18 & 0.009 \\
\hline Below knee rise time & 0.13 & -0.10 & -0.11 & -0.22 \\
\hline Lower thigh rise time & 0.11 & -0.08 & -0.12 & 0.11 \\
\hline
\end{tabular}

acid, the ribonucleoprotein complex, ribonucleic acid-binding protein LA, ribonucleoprotein, ribonucleic acid molecule/protein complex Sm, complement $\mathrm{C} 1$ subunit $\mathrm{C} 1 \mathrm{q}$, and phospholipids, and the dysfunction of $\mathrm{T}$ and $\mathrm{B}$ lymphocytes and dendritic cells. ${ }^{10}$

Atherosclerosis is a chronic inflammatory disease of the arteries associated with various risk factors that promote lipid abnormalities, development and progression of atherosclerotic lesions, plaque rupture, and vascular thrombosis ${ }^{11}$ Atherosclerosis is enhanced in autoimmune diseases. The reason for this accelerated process is still debatable and, although traditional risk factors (such as hyperlipidemia, smoking, obesity, hypertension, diabetes mellitus, postmenopausal status, and sedentary lifestyle) are more prevalent in those patients than in general population, they do not seem to fully explain that enhanced risk. ${ }^{12}$

Dyslipidemia with low high density lipoprotein, raised triglycerides, normal or mild elevation of LDL and raised lipoprotein is typically found in SLE, particularly in active disease. ${ }^{13}$ In our study, we found a statistically significant difference between patients and controls in serum cholesterol and LDL levels. These findings suggest that lupus is one of the etiologies of secondary dyslipidemia.

\begin{tabular}{lcc} 
Table 4. Correlation between intima media thickness versus other variables \\
\hline Variables & \multicolumn{2}{c}{ Intima media thickness } \\
\cline { 2 - 3 } & $p$ & $r$ \\
\hline Age & $<0.05 \mathrm{HS}$ & 0.42 \\
Systemic lupus erythematosus disease activity index & $<0.05 \mathrm{HS}$ & 0.28 \\
Disease duration & $>0.05$ & -0.012 \\
Erythrocyte sedimentation rate & $>0.05$ & 0.13 \\
Cholesterol & $>0.05$ & 0.14 \\
High density lipoprotein & $>0.05$ & -0.08 \\
Low density lipoprotein & $>0.05$ & 0.13 \\
Triglyceride & $>0.05$ & 0.22 \\
Cluster of differentiation 146 & $>0.05$ & 0.04 \\
Complement 3 & $>0.05$ & -0.08 \\
Complement 4 & $>0.05$ & -0.03 \\
Urine albumin and albumin/creatinine ratio & $<0.05 \mathrm{HS}$ & 0.33 \\
Brachial femoral pulse wave velocity & $>0.05$ & 0.13 \\
Brachial lower thigh pulse wave velocity & $>0.05$ & 0.22 \\
Brachial ankle pulse wave velocity & $>0.05$ & 0.12 \\
\hline HS: Highly significant. & & \\
\hline
\end{tabular}


Table 5. Correlation between systolic blood pressure and different lower limb segmental pulse wave velocities

\begin{tabular}{lc}
\hline & $\begin{array}{c}\text { Systolic blood } \\
\text { pressure }\end{array}$ \\
\hline Brachial femoral pulse wave velocity & $>0.05$ \\
Brachial lower thigh pulse wave velocity & $<0.05$ \\
Brachial below knee pulse wave velocity & $<0.05$ \\
Brachial ankle pulse wave velocity & $<0.05$ \\
\hline
\end{tabular}

Endothelial dysfunction was assessed by measurement of CD146 by flow cytometry while arterial stiffness was assessed by PWV. Our results showed a statistically significant difference in CD146 level between SLE patients and controls with a mean of $4.2 \pm 1.1,2.2 \pm 0.7$, respectively, and this in agreement with results of Robak et al. ${ }^{14}$ In contrast to our data, two other reports showed significant deficiency of circulating endothelial cell count in their SLE patients. ${ }^{15,16}$ An explanation for these discrepancies may be the fact that our study included Egyptians only, whereas their studies involved Africans, Americans, whites, and others. Our results showed no statistically significant correlation between CD146 and patients age or the disease duration, and this was consistent with findings of Elshal et al. ${ }^{17}$ and Abdelaziz et al., ${ }^{18}$ who demonstrated that CD146 level was independent of the age of SLE patients and/or the duration of the disease.

Our results showed a statistically significant positive correlation between CD146 and antinuclear antibodies $(\mathrm{p}<0.05)$ and anti-deoxyribonucleic acid (DNA) $(p<0.05)$, which was consistent with Elshal et al. ${ }^{17}$ who found significant positive correlation between CD146 and anti-DNA titer. However, our results were not consistent with Yao et al., ${ }^{19}$ who found no significant correlation between CD146 and anti-DNA.

Moreover, our findings agreed with Yao et al., ${ }^{19}$ who showed no significant correlation between CD146 and SLEDAI score, while disagreeing with Elshal et al. ${ }^{17}$ who detected positive correlation between CD146 and SLEDAI score. This may be explained by our patients having lower SLEDAI scores (mean 4.2) than scores of patients in their study (mean 18.75).

We found no significant correlation between lipid parameters and CD146 level and this is in consistency with the findings of Rajagopalan et al., ${ }^{20}$ who concluded that CD146+ in the peripheral circulation in SLE specifically correlated with vascular dysfunction in a cohort of young subjects with SLE, with low Framingham risk scores (score $<1 \%$ ), and independent of other traditional predictors of atherosclerosis, which may potentially indicate an additional mechanism for the predisposition to premature vascular disease.

In our study, there was no statistically significant correlation between complements C3, C4, and CD146 level and this is consistent with results of Yao et al. ${ }^{19}$ However, Kassem et al. ${ }^{21}$ showed a significant correlation between CD146 and low complement level. In this study, there was no statistically significant difference between SLE and controls in terms of IMT with a $p$ value above 0.05 . This was in accordance with findings of Nienhuis et al. ${ }^{22}$ who indicated that mean IMT did not differ between SLE patients and healthy controls. In addition, there was no statistically significant correlation between serum cholesterol level, LDL level, and IMT ( $p>0.05)$. This was in agreement with de Leeuw et al. ${ }^{23}$ who concluded that sex, body mass index, smoking, lipid levels, diabetes mellitus, family history for cardiovascular disease, levels of other endothelial activation markers, and medication did not correlate to IMT. These results raised the need for earlier markers of atherosclerosis since IMT does not appear to change early with several risk factors of atherosclerosis in SLE.

Furthermore, our results was in accordance with the study of Cacciapaglia et al. ${ }^{24}$ where IMT in patients with SLE significantly correlated with disease duration, and SLEDAI. However, our results differed from theirs since, in our study, there was no statistically significant relation between disease duration and carotid IMT. This may be due to the different features of patients, as in their study, the disease duration was longer with a mean of $29 \pm 13$ years; however, our patients had shorter disease duration with a mean of $1.5 \pm 1$ years.

Also, in our study, there was a statistically significant correlation between SLEDAI scores and brachial-femoral PWV, brachial-lower thigh PWV, and carotid IMT with no correlation to lipid profile.

Arterial stiffness increases in accelerated atherosclerosis due to inflammation. ${ }^{25}$ Large 
artery stiffness is recognized as a modifiable, independent predictor of cardiovascular risk. ${ }^{26}$ Structural components within the arterial wall, mainly collagen and elastin, together with transmural pressure, are key determinants of large arterial PWV. Increased circulating inflammatory mediators promote white cell infiltration into arteries, and a change in vascular smooth muscle phenotype. Both these cell types release the matrix metalloproteinases that can degrade elastin. Decrease of elastin from the media may be expected to result in arterial stiffening. Under inflammatory conditions, vascular smooth muscle cells also express osteoblast markers and can take up phosphate to produce bioapatite, leading to medial calcification and reduced arterial distensibility. Perivascular inflammation and cellular infiltration around the vasa vasorum may lead to vessel ischemia, which may also promote matrix remodeling and eventually stiffening of the arteries. ${ }^{27}$ Arteries become wider and less elastic (increased PWV). ${ }^{28}$

To our knowledge, this is the first study which measured PWV and RT simultaneously at four segments along the lower limb arterial tree to assess arterial stiffness in large and small arteries.

In our study, we found no statistically significant correlation between patients and controls in terms of PWV (brachial-femoral, brachial-lower thigh, brachial-below knee, brachial-ankle). This may be due to short disease duration of our SLE patients; however, the values were higher in the patients than controls.

Our results showed a statistically significant difference between patients and controls regarding femoral $\mathrm{RT}(\mathrm{p}<0.05)$ with patients having a mean of $39.3 \pm 7.2 \mathrm{RT}$ and controls having a mean of $45.7 \pm 7.4 \mathrm{RT}$. Also, there was a statistically significant difference between patients and controls in terms of lower thigh RT (lower femoral and proximal popliteal) with patients having a mean of $41 \pm 7$ RT and controls having a mean of $46.7 \pm 8$ RT. We also found a highly statistically significant difference between patients and controls in terms of ankle RT $(p<0.001)$ with patients having a mean of $31.9 \pm 6 \mathrm{RT}$ and controls having a mean of $36.9 \pm 8 \mathrm{RT}$.
A short RT indicates stiff non-compliant arteries which reach the peak of the waveform earlier than healthy arteries. It represents the active part of the waveform. This marker of arterial stiffness was increased in our SLE patients who had no history of cardiovascular disease and organ damage when compared to healthy controls, as our SLE patients had short RT compared to controls, this highlights the significance of RT in SLE patients as a predictor of arterial stiffness.

Intima media thickness of our patients showed no statistically significant difference when compared to controls $(p>0.05)$. This denotes that SLE vascular affection begins by vascular stiffness before overt atherosclerosis as most of our patients had normal IMT and normal ankle brachial index. This stiffness is evident in both the femoral arteries and ankle arteries and is more pronounced in the latter indicating earlier affection of peripheral arteries before central arteries. Our findings revealed that peripheral artery elasticity is decreased in SLE without increased IMT, and this is consistent with results published by Nienhuis et al. ${ }^{22}$ who concluded that vascular dysfunction, as shown by decreased small arterial elasticity, is present in quiescent SLE patients without atherosclerosis, and that this decrease is independent of traditional cardiovascular risk factors, but is related to the presence of SLE. Although IMT values were normal in our study, they correlated with the brachial-below knee PWV indicating early patchy atherosclerotic peripheral affection.

Accumulating data suggest an increased prevalence of arterial stiffness in SLE. Many factors play a role in the loss of arterial elasticity in this population, including both emerging and established vascular risk factors. Thus, arterial stiffness may be a useful index for risk stratification in SLE and has the potential to guide therapeutic decisions. ${ }^{29}$

Moreover, we detected a statistically significant correlation between brachial-femoral PWV and ankle PWV with serum $\mathrm{C} 3$ and $\mathrm{A} / \mathrm{C}$ ratio, and this correlation highlights the relation of proteinuria to premature atherosclerosis. There was a statistically significant correlation between brachial-femoral PWV, brachial-lower thigh PWV, and brachial-below knee brachial PWV 
with SLEDAI, possibly signifying the correlation between disease activity and arterial stiffness, and the importance of disease activity control to delay premature atherosclerosis.

Also, we demonstrated a statistically significant correlation between brachial-femoral PWV and serum LDLc. Furthermore, there was a statistically significant correlation between brachial-lower thigh PWV and brachial-below knee PWV with LDL and serum triglyceride $(p<0.05)$. IMT was not significantly changed yet with lipid profile in these patients ( $p>0.05)$, and this might indicate that $\mathrm{PWV}$ is an earlier indicator for lipid effect on the blood vessels compared to IMT, which renders PWV more valuable in the detection of early vascular affection in SLE and disease outcome.

Our results show a statistically significant positive correlation between brachial-lower thigh PWV, brachial-below knee PWV, and brachial-ankle PWV with systolic blood pressure $(p<0.05)$. This was in agreement with findings of Li et al., ${ }^{30}$ who concluded that brachial-ankle PWV was significantly correlated with systolic blood pressure and indicates arterial stiffness.

Brachial-femoral PWV is also a better positive predictor of vascular abnormalities with sensitivity of $71 \%$, specificity of $63 \%$, positive predictive value of $80 \%$, negative predictor value of $78 \%$, and an area under the receiver's operating characteristic curve of 0.6 .

In conclusion, elevated CD146 level is a feature of SLE, and CD146 and brachial-femoral PWV are useful early markers of vascular affection in SLE compared to increased IMT. PWV correlates with the lipid profile and SLE vascular affection is more pronounced in small arteries. Moreover, RT is a useful marker of arterial stiffness; however, further studies with larger sample size are required to support these findings.

\section{Declaration of conflicting interests}

The authors declared no conflicts of interest with respect to the authorship and/or publication of this article.

\section{Funding}

The authors received no financial support for the research and/or authorship of this article.

\section{REFERENCES}

1. Barbulescu AL, Vreju F, Cojocaru-Gofita IR, Musetescu $\mathrm{AE}$, Ciurea PL. Impaired arterial stiffness in systemic lupus ertythematosus - correlations with inflammation markers. Curr Health Sci J 2012;38:61-5.

2. Symmons DP, Gabriel SE. Epidemiology of CVD in rheumatic disease, with a focus on RA and SLE. Nat Rev Rheumatol 2011;7:399-408.

3. Hak AE, Karlson EW, Feskanich D, Stampfer MJ, Costenbader $\mathrm{KH}$. Systemic lupus erythematosus and the risk of cardiovascular disease: results from the nurses' health study. Arthritis Rheum 2009;61:1396-402.

4. Tziomalos K, Tziomalos K, Sivanadarajah N, Mikhailidis DP, Boumpas DT, Seifalian AM. Increased risk of vascular events in systemic lupus erythematosus: is arterial stiffness a predictor of vascular risk? Clin Exp Rheumatol 2008;26:1134-45.

5. Laurent S, Cockcroft J, Van Bortel L, Boutouyrie P, Giannattasio C, Hayoz D, et al. Expert consensus document on arterial stiffness: methodological issues and clinical applications. Eur Heart J 2006;27:2588-605.

6. Tanaka H, Munakata M, Kawano Y, Ohishi M, Shoji T, Sugawara J, et al. Comparison between carotid-femoral and brachial-ankle pulse wave velocity as measures of arterial stiffness. J Hypertens 2009;27:2022-7.

7. Harhouri K, Kebir A, Guillet B, Foucault-Bertaud A, Voytenko S, Piercecchi-Marti MD, et al. Soluble CD146 displays angiogenic properties and promotes neovascularization in experimental hind-limb ischemia. Blood 2010;115:3843-51.

8. Younis Hosni A, Tony Effat AE, Amin Nabila F. Serum CD 146, as a marker of endothelial dysfunction in chronic renal failure: a comparison between hemodialyzed and non-dialyzed patients. MMJ 2011;24:119-30.

9. Hochberg MC. Updating the American College of Rheumatology revised criteria for the classification of systemic lupus erythematosus. Arthritis Rheum 1997;40:1725.

10. D'Cruz DP, Khamashta MA, Hughes GR. Systemic lupus erythematosus. Lancet 2007;369:587-96.

11. Bassi N, Ghirardello A, Iaccarino L, Zampieri S, Rampudda ME, Atzeni F, et al. OxLDL/beta2GPIanti-oxLDL/beta2GPI complex and atherosclerosis in SLE patients. Autoimmun Rev 2007;7:52-8.

12. Matsuura E, Kobayashi K, Lopez LR. Preventing autoimmune and infection triggered atherosclerosis for an enduring healthful lifestyle. Autoimmun Rev 2008;7:214-22.

13. Mikdashi J, Handwerger B, Langenberg P, Miller M, Kittner S. Baseline disease activity, hyperlipidemia, and hypertension are predictive factors for ischemic stroke and stroke severity in systemic lupus erythematosus. Stroke 2007;38:281-5. 
14. Robak E, Kierstan M, Cebula B, Krawczynska A, Sysa-Jedrzejowska A, Wierzbowska A, et al. Circulating endothelial cells and angiogenic proteins in patients with systemic lupus erythematosus. Lupus 2009;18:332-41.

15. Lee PY, Li Y, Richards HB, Chan FS, Zhuang H, Narain S, et al. Type I interferon as a novel risk factor for endothelial progenitor cell depletion and endothelial dysfunction in systemic lupus erythematosus. Arthritis Rheum 2007;56:3759-69.

16. WesterweelPE, Luyten RK, Koomans HA, DerksenRH, Verhaar MC. Premature atherosclerotic cardiovascular disease in systemic lupus erythematosus. Arthritis Rheum 2007;56:1384-96.

17. Elshal M, Abdelaziz A, Abbas A, Mahmoud K, Fathy $\mathrm{H}$, El Mongy S, et al. Quantification of circulating endothelial cells in peripheral blood of systemic lupus erythematosus patients: a simple and reproducible method of assessing endothelial injury and repair. Nephrol Dial Transplant 2009;24:1495-9.

18. Abdelaziz Abeer M, Elshal Mohamed F, Fathy $\mathrm{H}$, Mongy SE, El-Basyuoni SR, Sayed Ahmed HMM, et al. Circulating endothelial cells in systemic lupus erythematosus: correlation with the activity of the disease. J Egypt worn Dermatol Soc.2008;5:37-8.

19. Yao G, Liu ZH, Zheng C, Zhang X, Chen H, Zeng $\mathrm{C}$, et al. Evaluation of renal vascular lesions using circulating endothelial cells in patients with lupus nephritis. Rheumatology (Oxford) 2008;47:432-6.

20. Rajagopalan S, Somers EC, Brook RD, Kehrer C, Pfenninger D, Lewis E, et al. Endothelial cell apoptosis in systemic lupus erythematosus: a common pathway for abnormal vascular function and thrombosis propensity. Blood 2004;103:3677-83.

21. Kassem E, El-Sergany M, El-Saadany H, Shahba A, Salah W. Circulating endothelial cells and cardiovascular risk in systemic lupus erythematosus, J Am Sci 2010;6:700-7.
22. Nienhuis HL, de Leeuw K, Bijzet J, van Doormaal JJ, van Roon AM, Smit AJ, et al. Small artery elasticity is decreased in patients with systemic lupus erythematosus without increased intima media thickness. Arthritis Res Ther 2010;12:181.

23. de Leeuw K, Smit AJ, de Groot E, van Roon AM, Kallenberg CG, Bijl M. Longitudinal study on premature atherosclerosis in patients with systemic lupus erythematosus. Atherosclerosis 2009;206:546-50.

24. Cacciapaglia F, Zardi EM, Coppolino G, Buzzulini F, Margiotta D, Arcarese L, et al. Stiffness parameters, intima-media thickness and early atherosclerosis in systemic lupus erythematosus patients. Lupus 2009; 18:249-56.

25. Wang YX, Fitch RM. Vascular stiffness: measurements, mechanisms and implications. Curr Vasc Pharmacol 2004;2:379-84.

26. Laurent S, Boutouyrie P, Asmar R, Gautier I, Laloux $\mathrm{B}$, Guize L, et al. Aortic stiffness is an independent predictor of all-cause and cardiovascular mortality in hypertensive patients. Hypertension 2001;37:1236-41.

27. Mackenzie IS, Wilkinson IB, Cockcroft JR. Assessment of arterial stiffness in clinical practice. QJM 2002;95:67-74.

28. Learoyd BM, Taylor MG. Alterations with age in the viscoelastic properties of human arterial walls. Circ Res 1966;18:278-92.

29. Tziomalos K, Tziomalos K, Sivanadarajah N, Mikhailidis DP, Boumpas DT, Seifalian AM. Increased risk of vascular events in systemic lupus erythematosus: is arterial stiffness a predictor of vascular risk? Clin Exp Rheumatol 2008;26:1134-45.

30. Li B, Gao H, Li X, Liu Y, Wang M. Correlation between brachial-ankle pulse wave velocity and arterial compliance and cardiovascular risk factors in elderly patients with arteriosclerosis. Hypertens Res 2006;29:309-14. 